\title{
THE DECOMPOSITION OF ARTINIAN MODULES OVER HYPER-(CYCLIC OR FINITE) GROUPS
}

\author{
by Y. B. QIN \\ (Received 28th June 1994)
}

\begin{abstract}
If $G$ is a hyperfinite locally soluble group and $A$ an artinian $Z G$-module then Zaicev proved that $A$ has an $f$ decomposition. For $G$ being a hyper-(cyclic or finite) locally soluble group, $Z$. Y. Duan has shown that any periodic artinian ZG-module $A$ has an $f$-decomposition. Here we prove that: if $G$ is a hyper-(cyclic or finite) group, then any artinian $Z G$-module $A$ has an $f$-decomposition.
\end{abstract}

1991 Mathematics subject classification: $20 \mathrm{~F} 19$

In 1986, D. I. Zaicev [7] proved that if $G$ is a hyperfinite locally soluble group, then any artinian $Z G$-module $A$ has an $f$-decomposition. Also in 1991, Z. Y. Duan [2] proved that any periodic artinian module over a hyper-(cyclic or finite) locally soluble group has an $f$-decomposition. In this paper, we consider artinian modules over hyper(cyclic or finite) groups and generalize these two results. That is

Theorem. If $G$ is a hyper-(cyclic or finite) group, then any artinian $\mathbf{Z G - m o d u l e ~} A$ has an $f$-decomposition.

Our proof of the above theorem depends on the results in [1] and is similar with that of Theorem 1 in [2]. First, we prove the following two lemmas which are necessary for the proof of the theorem.

Lemma 1. Any finitely generated hyper-(cyclic or finite) group is a supersoluble-byfinite group.

Proof. Let $1=G_{0} \triangleleft G_{1} \triangleleft \ldots \triangleleft G_{\alpha}=G$ be an ascending normal series of subgroups of a finitely generated group $G$ in which each factor $G_{\beta+1} / G_{\beta}$ is cyclic or finite and furthermore we may assume that if $G_{\beta+1} / G_{\beta}$ is finite, then there is no subgroup $K$ such that $K$ is normal in $G$ and $G_{\beta}<K<G_{\beta+1}$. Since $G / G_{\beta}=1$ is clearly a supersoluble-byfinite group, we may assume that there exists $\beta \leq \alpha$ such that $G / G_{\beta}$ is supersoluble-by- 
finite but $G / G_{y}$ is not for all $\gamma<\beta$. We claim that $\beta=0$. For otherwise, if $\beta-1$ exists, then we consider the following two cases:

(i) $G_{\beta} / G_{\beta-1}$ is cyclic. Clearly $G / G_{\beta-1}$ is supersoluble-by-finite, a contradiction.

(ii) $G_{\beta} / G_{\beta-1}$ is finite. If $G_{\beta} / G_{\beta-1} \leq C_{G / G_{\beta-1}}\left(G_{\beta} / G_{\beta-1}\right)$, then $G_{\beta} / G_{\beta-1}$ is abelian. Since $G / G_{\beta}$ is supersoluble-by-finite, there is an $H$ such that $G / H$ is finite and $H / G_{\beta}$ is supersoluble. By $G_{\beta} / G_{\beta-1}$ being finite and abelian, $H / G_{\beta-1}$ is polycyclic and so is residually finite, therefore there is an $N$ with $H / N$ finite and $N \cap G_{\beta}=G_{\beta-1}$. Thus $N / G_{\beta-1} \cong N G_{\beta} / G_{\beta}$ is supersoluble and so $G / G_{\beta-1}$ is supersoluble-by-finite, a contradiction. Thus $\left(G_{\beta} / G_{\beta-1}\right) \cap C_{G / G_{\beta-1}}\left(G_{\beta} / G_{\beta-1}\right)=1$. Let $K / G_{\beta-1}=C_{G / G_{\beta-1}}\left(G_{\beta} / G_{\beta-1}\right)$, then $G / K$ is finite and $K \cap G_{\beta}=G_{\beta-1}$. Thus $K / G_{\beta-1} \cong K G_{\beta} / G_{\beta}$ is supersoluble-by-finite and so is $G / G_{\beta-1}$, a contradiction again. Thus $\beta-1$ does not exist, i.e. $\beta$ is a limit ordinal. Since $G / G_{\beta}$ is finitely generated, by $\left[5\right.$, p. 403], $G_{\beta}$ is finitely generated as a $G$-operator group. Let $G_{\beta}=\left\langle x_{1}, \ldots, x_{n}\right\rangle^{G}$ and since $G_{\beta}=\bigcup_{\alpha>\beta} G_{\gamma}$ so there exist $\gamma_{1} \ldots \gamma_{n}$ such that $x_{i} \in G_{\gamma_{i}}$. Let $\gamma_{0}<\beta$ such that $\gamma_{i}<\gamma_{0}$ for all $i=0, \ldots, n$, then $x_{i} \in G_{\gamma_{0}}$ for all $i$. Since $G_{\gamma_{0}} \triangleleft G$, so $G_{\beta}=\left\langle x_{1}, \ldots, x_{n}\right\rangle^{G} \leq\left\langle G_{\gamma_{0}}\right\rangle^{G}=G_{\gamma_{0}}$. Thus $G / G_{\beta}=G / G_{\gamma_{0}}$, contrary to the hypothesis for $\beta$. Hence $\beta=0$ and then the result is proved.

The above lemma is necessary for removing the locally soluble condition and in fact, the following lemma is the beginning of the proof of the theorem.

Lemma 2. Let $G$ be a hyper-(cyclic or finite) group, $B$ an artinian $\mathbf{Z} G$-module and $A$ a $Z G$-submodule of $B$ such that all irreducible $Z G$-factors of $A$ are infinite. If $B / A$ is finite, Then $B=A \oplus B_{1}$ for some $\mathbf{Z} G$-submodule $B_{1}$ of $B$.

Proof. Since $A$ is artinian, it is possible to choose a $\mathrm{ZG}$-submodule $B_{1}$ of $B$ such that $B=A+B_{1}$ and for each $U \leq B$ with $B=A+U$, the intersection $A \cap U$ and $A \cap B_{1}$ are equal. We prove that $A \cap B_{1}=0$.

Suppose $A_{1}=A \cap B_{1} \neq 0$ and assume that $G$ acts faithfully on $B_{1}$. Since $B / A \cong{ }_{\mathrm{zG}} B_{1} / A_{1}$, then $B_{1} / A_{1}$ is finite. Let $G_{1}=C_{G}\left(B_{1} / A_{1}\right)$, then $G_{1}$ contains a normal subgroup $K$ of $G$ which is either finite or cyclic. Let $H=G_{1} \cap C_{G}(K)$, then $G / H$ is finite. Let $1 \neq x \in K$, then $H \leq C_{G}(x) \leq G$. Consider $B_{1}, A_{1}$ as $Z H$-modules. By Lemma 3 in [3], $B_{1}(x-1)$ and $C_{B_{1}}(x)$ are $\mathrm{Z} H$-submodules of $B_{1}$ and $B_{1}(x-1) \cong{ }_{\mathrm{Z} H} B_{1} / C_{B_{1}}(x)$. Since $A_{1}<A$ and all irreducible $\mathbf{Z} G$-factors of $A_{1}$ are infinite, then so are all irreducible $\mathbf{Z} H$-factors of $A_{1}$. Since $x \in C_{G}\left(B_{1} / A_{1}\right)$, then $B_{1}(x-1)<A_{1}$ and so $B_{1}(x-1)=\left(B_{1}(x-1)\right)^{f}$. On the other hand, define $\varphi: B_{1} / A_{1} \rightarrow B_{1}(x-1) / A_{1}(x-1)$ such that $\varphi\left(b+A_{1}\right)=b(x-1)+A_{1}(x-1)$, where $b \in B_{1}$. Clearly $\varphi$ is a homomorphism from $B_{1} / A_{1}$ to $B_{1}(x-1) / A_{1}(x-1)$. Since $B_{1} / A_{1}$ is finite, then $B_{1}(x-1) / A_{1}(x-1)$ is finite, contrary to $B_{1}(x-1)=\left(B_{1}(x-1)\right)^{f}$. So $B_{1}(x-1)=A_{1}(x-1)$, and then $B_{1}=A_{1}+C_{B_{1}}(x)$. Since $B=A+B_{1}=A_{1}+C_{B_{1}}(x)+A=$ $A+C_{B_{1}}(x)$, then $A \cap C_{B_{1}}(x)<A \cap B_{1}$ which is contrary to the choice of $B_{1}$. So $A \cap B_{1}=0$ and the lemma is proved.

Now we can prove the theorem completely. 
Theorem. If $G$ is a hyper-(cyclic or finite) group, then any artinian ZG-module $A$ has an $f$-decomposition.

Proof. Suppose that $A$ does not have an $f$-decomposition, then we can find a submodule $A_{1}$ not having an $f$-decomposition such that every proper submodule of $A_{1}$ does have an $f$-decomposition. We may assume that $A$ satisfies this condition, i.e., $A=A_{1}$, and further assume that $G$ acts faithfully on $A$. It follows that $A$ is not a sum of proper submodules and so $A$ has a unique maximal submodule $M$ containing every proper submodule of $A$. For each $a \in A-M,\langle a\rangle^{G}=A$. If $G$ were finite, then the abelian group $A$ would be finitely generated and since $A$ is artinian, then $A$ is finite, a contradiction. So $G$ is infinite.

Let $M=M^{\delta} \oplus M^{\bar{s}}$ be the $f$-decomposition of $M$. We consider the following two cases $A / M$ is finite or infinite. If $A / M$ is finite, then $M^{\bar{f}} \neq 0$. For otherwise, for any irreducible $\mathrm{Z} G$-factor $C / D$ of $A$, if $C \leq M$, then $C / D$ is finite since $M=M^{f}$; if $C>M$, then $C=A$ and so $D=M$ since $M$ is the unique maximal submodule of $A$ and $C / D$ is irreducible, contrary to $A \neq A^{f}$. Consider $A / M^{f}$. By Lemma 2, $A / M^{f}=M / M^{f} \oplus B / M^{f}$ for some $\mathrm{Z} G$-submodule $B$ of $A$, then $A=M+B<M$ since $M$ contains all proper $Z G$-submodules of $A$, a contradiction. Thus $A / M$ is infinite. Also $M^{f} \neq 0$, for otherwise we can get a contradiction using the same method as above. Considering $A / M^{f}$, we may assume that $M^{f}=0$ and so $M=M^{\vec{s}}$.

Let $D$ be a finite $\mathrm{Z} G$-submodule of $M$ and $H=C_{G}(D)$, then $G / H$ is finite, $H$ contains a nontrivial infinite cyclic or finite subgroup $K$ normal in $G$. Let $G_{1}=C_{G}(K)$, then $G / G_{1}$ is finite. By Corollary 2.2 .2 in [1], $A / M$ contains an infinite irreducible $Z G$-submodule $A_{1}^{*} / M$ and also by Lemma 2.2 .4 in $[1], A / M=D r_{s \in S}\left(A_{1}^{*} / M\right) s$, where $S$ is a subset of transversal $T$ to $G_{1}$ in $G$. Thus $A / M$ is a direct sum of finitely many irreducible $Z G_{1}$-submodules. Let $A / M=A_{1}^{*} / M \oplus A_{2}^{*} / M \oplus \ldots \oplus A_{n}^{*} / M$. Let $x \in K, x \neq 1$, then $G_{1}=$ $C_{G}(K) \leq C_{G}(x) \leq G$ and so $A_{i}^{*}(x-1)$ and $C_{A_{i}^{*}}(x)$ are both $Z G$-submodules of $A_{i}^{*}$ for each $i$. Now we can choose some $i \in\{1, \ldots, n\}$ such that $x$ acts nontrivially on $A_{i}^{*}$. For otherwise, if $x$ acts trivially on $A_{i}^{*}$ for each $i$, then $x$ acts trivially on $A$, contrary to $G$ acting trivially on $A$. For $A_{i}^{*}$, it is clear that: (a) $A_{i}^{*}$ does not have an $f\left(Z G_{1}\right)$-decomposition and $C_{A^{*}}(x) \neq A_{i}^{*}$, (b) $M$ is the unique maximal $Z G_{1}$-submodule of $A_{i}^{*}$ with $M=M^{f}$; (c) $A_{i}^{*} / M$ is infinite and for each $a \in A_{i}^{*}-M, A_{i}^{*}=\langle a\rangle^{G_{1}}$. Now we consider the following two cases:

(1) If $A_{i}^{*}(x-1)<A_{i}^{*}$, then $A_{i}^{*}(x-1)<M$. For $\varphi: a+M \rightarrow a(x-1)+M(x-1)\left(a \in A_{i}^{*}\right)$, we have $A_{i}^{*} / M_{Z G_{1}}^{\mathscr{Q}} A_{i}^{*}(x-1) / M(x-1)$ and $\operatorname{Ker} \varphi=0$ or $A_{i}^{*} / M$. If $\operatorname{Ker} \varphi=0$, then $A_{i}^{*}(x-1) / M(x-1)$ is an infinite irreducible $Z G_{1}$-factor of $M$, a contradiction. So $\operatorname{Ker} \varphi=A_{i}^{*} / M$. That is $A_{i}^{*}(x-1)=M(x-1)$ and then $A_{i}^{*}=M+C_{A_{j}}(x)$. Since $C_{A_{i}^{*}}(x) \neq A_{i}^{*}$, then $C_{A_{i}^{*}}(x)<M$ and $A_{i}^{*}=M+C_{A_{i}^{*}}(x)=M<A_{i}^{*}$, a contradiction again.

(2) If $A_{i}^{*}(x-1)=A_{i}^{*}$, so for $a \in A_{i}^{*}-M$, there exists $a_{0} \in A_{i}^{*}$ such that $a=a_{0}(x-1)$ and $A_{i}^{*}=\langle a\rangle^{G_{1}}$. Choose a finitely generated subgroup $L^{*}$ of $G_{1}$ such that $a_{0 ;} \in\langle a\rangle^{L^{*}}$, $D \leq\langle a\rangle^{L}$. Let $L=\left\langle L^{*}, x\right\rangle$ and $A_{1}=\langle a\rangle^{L}$, then $A_{1}$ is a finitely generated $\mathbf{Z} L$-module and $L$ is a finitely generated hyper-(cyclic or finite) group. By Lemma $1, L$ is a supersolubleby-finite group. Thus $A_{1}$ has a $Z L$-submodule $B_{1}$ of finite index such that $D \cap B_{1} \neq D$ by the residual finiteness of finitely generated abelian-by-polycyclic groups [4]. Consider 
the finite $Z L$-module $A_{1} / B_{1}$. Since $G_{1}=C_{G}(K) \leq C_{G}(x)$ and so $\left[G_{1}, x\right]=1$, we have $\left[L^{*}, x\right]=1$ and then $x \in Z(L)$ since $L=\left\langle L^{*}, x\right\rangle$. Thus $A_{1} / B_{1}$ can be viewed as a $Z\langle x\rangle$-module. Then, by [6], we can get $A_{1} / B_{1}=B / B_{1} \oplus C / B_{1}$, where the $Z\langle x\rangle$ submodule $B / B_{1}$ has a $Z\langle x\rangle$-composition series in which each $Z\langle x\rangle$-factor is $\langle x\rangle$-trivial and the $Z\langle x\rangle$-submodule $C / B_{1}$ has no nonzero $Z\langle x\rangle$-factors which are $\langle x\rangle$-trivial. Since $\left(D+B_{1}\right) / B_{1}$ is an $x$-trivial $Z\langle x\rangle$-submodule of $A_{1} / B_{1}$, so $B / B_{1} \neq 0$. Thus $A_{1} / C$ is a nonzero finite $Z\langle x\rangle$-module and $A_{1} / C \cong{ }_{Z\langle x\rangle}\left(A_{1} / B_{1}\right) /\left(C / B_{1}\right) \cong{ }_{Z\langle x\rangle} B / B_{1}$ shows that $A_{1} / C$ has a finite $\mathbf{Z}\langle x\rangle$-composition series in which each $\mathbf{Z}\langle x\rangle$-factor is $\langle x\rangle$-trivial. Hence $\left(A_{1}(x-1)+C\right) / C=\bar{A}_{1}(x-1)<\bar{A}_{1}$, where $\bar{A}_{1}=A_{1} / C$. But on the other hand, since $A_{1}(x-1)$ is a $Z L$-module and $a_{0} \in A_{1}$, so

$$
A_{1}=\langle a\rangle^{L}=\left\langle a_{0}(x-1)\right\rangle^{L}=\left(\left\langle a_{0}\right\rangle(x-1)\right)^{L} \leq\left(A_{1}(x-1)\right)^{L}=A_{1}(x-1)
$$

a contradiction. This finishes the proof of the theorem.

This paper is directed by the author's supervisor, Dr. Z. Y. Duan, and the author would take this opportunity of expressing his deepest gratitude to him for his invaluable help and encouragement.

\section{REFERENCES}

1. Z. Y. DuAN, Noetherian modules over hyperfinite groups (Ph. D. Thesis, University of Glasgow, 1991).

2. Z. Y. Duan, Extensions of abelian by hyper-(cyclic or finite) groups 1, Comm. Algebra 20(8) (1992), 2305-2321.

3. Z. Y. DUAN, The $f$-decomposition of artinian modules over hyperfinite groups, to appear.

4. A. V. JalegaonKar, Integral group rings of polycyclic-by-finite groups, J. Pure, Appl. Alg. 4 (1974), 337-343.

5. D. J. S. Robinson, A Course in the Theory of Groups (Springer-Verlag, New York, 1982).

6. D. I. ZAICEv, Hypercyclic extensions of abelian groups, Akad. Nauk Ukrain. SSR, Inst. Mat. Kiev (1979), 16-37.

7. D. I. ZAICEv, Splitting of extensions of abelian groups, Akad. Nauk Ukrain. SSR, Inst. Mat. Kiev (1986), 21-31.

Department of Applying Mathematics

Southwest China Communication University

Chengdu 610031

P. R. China 